\title{
Acoustic Coupling Fluid
}

National Cancer Institute

\section{Source}

National Cancer Institute. Acoustic Coupling Fluid. NCI Thesaurus. Code C115100.

A brain mimicking fluid with an attenuation coefficient similar to that found in the adult human brain, which can potentially improve the quality of an image acquired during intraoperative ultrasonog raphy. Upon administration into the resection cavity during surgical removal of a brain tumor, the acoustic coupling fluid may both increase the quality of the ultrasound image and improve the visualization of the tumor. This may facilitate the surgical removal of residual tumor while sparing normal, healthy brain tissue. 